\title{
Airway Hyperresponsiveness to Mannitol in Obesity Before and After Bariatric Surgery
}

\author{
Ebymar Arismendi • Eva Rivas - Josep Vidal • Esther Barreiro • \\ Yolanda Torralba • Felip Burgos $•$ Roberto Rodriguez-Roisin
}

Published online: 25 January 2015

(C) The Author(s) 2015. This article is published with open access at Springerlink.com

\begin{abstract}
Background The relationship between airway hyperresponsiveness (AHR) and obesity, a low-grade systemic inflammatory condition, remains largely unknown. It is established that AHR to indirect stimuli is associated with active airway inflammation. The objectives were to
\end{abstract}

E. Arismendi $\cdot$ F. Burgos $\cdot$ R. Rodriguez-Roisin

Servei de Pneumologia (Institut del Tòrax), and Fundació Clínic per

la Recerca Biomèdica, Hospital Clínic, Barcelona, Spain

E. Rivas

Servei de Anestesiologia and Fundació Clínic per la Recerca

Biomèdica, Hospital Clínic, Barcelona, Spain

J. Vidal $\cdot$ F. Burgos $\cdot$ R. Rodriguez-Roisin

Institut d'Investigacions Biomédiques August Pi i Sunyer

(IDIBAPS), Universitat de Barcelona, Barcelona, Spain

E. Arismendi $\cdot$ E. Barreiro $\cdot$ F. Burgos $\cdot$ R. Rodriguez-Roisin

Ciber Enfermedades Respiratorias (CIBERES), Barcelona, Spain

J. Vidal

Servei de Endocrinologia, and Fundació Clínic per la Recerca

Biomèdica, Hospital Clínic, Barcelona, Spain

\section{E. Barreiro}

Pulmonology Department, Hospital del Mar, Parc de Recerca

Biomèdica de Barcelona (PRBB), Universitat Pompeu Fabra,

Barcelona, Spain

Y. Torralba

Servei de Pneumologia (Institut del Tòrax), and Fundació Clínic per la Recerca Biomèdica, Ciber Enfermedades Respiratorias

(CIBERES), Barcelona, Spain

R. Rodriguez-Roisin $(\square)$

Institut Clínic del Tòrax, Servei de Pneumologia, Hospital Clínic,

Villarroel, 170, Barcelona 08036, Spain

e-mail: rororo@clinic.ub.es

J. Vidal

Ciber Diabetes y Enfermedades Metabólicas (CIBERDEM),

Barcelona, Spain investigate the rate of AHR to mannitol in obese subjects and its changes 1 year after bariatric surgery (BS).

Methods We enrolled 58 candidates to BS severely obese (33 nonsmokers and 25 smokers) without history of asthma and 20 healthy, nonobese participants and related AHR to functional findings and serum and exhaled biomarkers.

Results Before surgery, AHR was observed in 16 (28\%) obese with the provocation doses of mannitol to induce a $15 \%$ fall in $\mathrm{FEV}_{1}\left(\mathrm{PD}_{15}\right)$ of (geometric mean [95\% CI]) 83 (24-145) mg. Compared to control participants, obese participants had lower spirometric values and higher serum and exhaled biomarkers ( $p<0.05$ each). After surgery, AHR was abolished $(p<0.01)$ in all but four obese subjects.

Conclusions Weight loss induced by BS was the key independent factor associated to AHR improvement. AHR to mannitol is highly prevalent in obesity, and it is largely abolished by BS.

Keywords Abdominal obesity - Airway inflammation . Bronchial hyperreactivity $\cdot$ Indirect bronchoconstrictor . Systemic inflammation

\section{Introduction}

The concurrence of obesity and bronchial asthma has become an increasing worldwide major public health problem $[1,2]$. Some studies show a clear evidence for a relation of airway hyperresponsiveness (AHR) to body mass index (BMI) [3]. However, the mechanisms of the relationship between obesity, AHR, and asthma are not sufficiently established [4, 5], and the impact of its underlying low-grade chronic systemic inflammation on AHR remains unsettled [6]. Obesity is characterized by an increased number of resident macrophages in adipose tissue that secrete a variety of inflammatory molecules, such as leptin and adiponectin [7, 8]. It is known that AHR to osmotic challenge agents is associated with the presence of airway inflammation as they increase airway 
osmolarity and induce the release of inflammatory cells and of mediators that act on their specific receptors on the airway smooth muscle causing contraction [9, 10].

Bariatric surgery (BS) is the most efficacious therapeutic strategy to achieve major and sustained weight loss in morbidly obese subjects [11] and is associated with improved systemic inflammation [12]. There have been very few studies in obese subjects, mostly in asthmatics, to assess AHR using methacholine [13] before and after effective weight loss with discrepant results [14-17]. We investigated the prevalence of AHR to mannitol in obese candidates to BS before and 1 year after BS. Part of the results of this study has been previously reported in abstract form [18].

\section{Methods}

\section{Subjects}

This is a prospective, observational study in 58 morbidly obese individuals (44 females/14 males; aged $46 \pm 12$ years) with $\mathrm{BMI} \geq 40 \mathrm{~kg} / \mathrm{m}^{2}$ or $\geq 35 \mathrm{~kg} / \mathrm{m}^{2}$ (in patients with obesityrelated diseases), candidates to $\mathrm{BS}$ in our center [18]. This sample of obese subjects was part of a study with a larger cohort of severe obese candidates to BS aimed to describe the systemic inflammome before and after BS [19]. Thirtythree obese individuals were nonsmokers ( $<10$ pack-years) and 25 current ( $\geq 10$ pack-years) or former ( $\geq 1$ year after complete cessation) smokers (38 \pm 26 pack-years). Participants with cardiovascular and pulmonary (i.e., asthma, chronic obstructive pulmonary disease, and bronchiectasis) diseases were excluded. One year after BS, participants were also evaluated. A control group of 20 healthy nonsmokers (16 females/4 males), with normal weight and lung function, age-matched (46 \pm 7 years) was also recruited. Written informed consent (Protocol 2008/4015) was obtained from each participant according to the requirements of the Ethics Committee of the Hospital Clínic, Universitat de Barcelona.

BS including either Roux-en-Y gastric bypass or sleeve gastrectomy was carried out according to standard procedures [20]. The selection of the surgical technique was based on the presence of larger BMI, an estimated operative risk, or the presence of an enlarged liver [21]. Postoperative weight loss was expressed as a percentage of the presurgical excess weight ( $\%$ excess weight loss $=[100 \times[$ weight before surgery - weight at the time of evaluation]/[weight before surgery-weight corresponding to body mass index $\left.=25 \mathrm{~kg} / \mathrm{m}^{2}\right]$ ) [20]. BS was considered successful when excess weight loss was $>50 \%$ of presurgical excess weight.
Study Design

The 58 enrolled obese subjects visited the laboratory twice on two consecutive days, before (median, 5 weeks) and 1 year after BS (median, 13 months). At day 1, clinical evaluation, forced spirometry, and static lung volumes using our own predicted values [22-25] were carried out; at day 2, serum and exhaled samples were obtained, and the mannitol challenge was performed in all obese participants. At day 1, serum and exhaled samples and forced spirometry were determined in all control individuals; at day 2 , eight subjects were challenged to mannitol alone. Control participants were explored once only.

\section{Mannitol Challenge}

Mannitol challenge was carried out using a commercially available kit (Pharmaxis Ltd, Burnham, UK) [26]. Bronchial challenge to mannitol was performed as previously reported by our group [27]. Pre-challenge baseline $\mathrm{FEV}_{1}$ was used to calculate the maximum \% fall $\mathrm{FEV}_{1}$ after mannitol. The dose of mannitol that induced a fall of $15 \%$ below baseline (i.e., $\mathrm{PD}_{15}$ ) was expressed as geometric mean $(\mathrm{GM})\left(\mathrm{GM} \mathrm{PD}_{15}\right)$ in milligrams. AHR to mannitol was defined as $\mathrm{PD}_{15}<635 \mathrm{mg}$. The responsedose ratio (RDR) was calculated as the $\%$ fall $F E V_{1}$ at the last dose, divided by the total cumulative dose of mannitol given (expressed as $\%$ fall $/ \mathrm{mg}$ ) in milligrams administered [26]. The test stopped when $15 \%$ fall $\mathrm{FEV}_{1}$ was achieved or the cumulative dose of $635 \mathrm{mg}$ had been administered. Mannitol bronchoprovocation was completed by control and obese individuals without major adverse events.

\section{Biomarker Measurements}

Serum and exhaled samples were obtained and stored at $-80{ }^{\circ} \mathrm{C}$. Serum concentrations of C-reactive protein (CRP) were determined using an immunoturbidimetry method (ADVIA Chemistry, Siemens Tarrytown, NY, USA) and those of leptin using the specific enzyme-linked immunoabsorbent assay (ELISA) kit (Diagnostic Biochem Canada Inc., Ontario, Canada). Serum adiponectin, soluble tumor necrosis factorreceptor 1(sTNF-R1), and interleukin (IL)-8 levels were determined using a specific ELISA (US Biological Salem, MA, USA; IBL international Hamburg, Germany; and Anogen Ontario, Canada), respectively. Exhaled breath condensate samples of IL-8 were determined using an EcoScreen condenser (Jaeger, Würzburg, Germany) following current recommendations [28, 29] and specific ELISA kits (Cayman Chemical Company, Ann Arbor, MI, USA).

All biomarker measurements were performed in duplicate and the mean value used for analysis. Biomarker concentrations were below the lower limit of quantification (LLQ) in 
some individuals. To avoid a downward bias of biomarkers, a nominal level of half of the LLQ value was used in the analysis in individuals with values below the LLQ [30].

\section{Statistical Analysis}

Data were expressed as mean $\pm \mathrm{SD}$ or median (interquartile range). Comparisons between obese and control individuals before BS were evaluated using one-way ANOVA with Bonferroni post hoc analysis (parametric) and KruskalWallis (nonparametric) test. For comparisons between obese subjects before and after surgery, paired Student's for related samples (parametric) or Wilcoxon (nonparametric) tests for continuous variables were used. Unpaired Student's (parametric) and Mann-Whitney $U$ (nonparametric) tests with Bonferroni-Holm correction were performed to compare obese individuals with and without AHR. Chi-square and $\mathrm{McNemar}$ tests for categorical variables and Spearman correlations were used as appropriate. An odds ratio calculated using generalized estimating equations (GEE) analysis [31], to account for non-independent evaluations from the same individuals before and after BS, was determined to assess the potential relationships of anthropometric data, cigarette smoking, and serum and exhaled biomarkers with AHR response. Statistical analysis was performed with specialized computer software (SPSS 20.0, Chicago, IL). Unless otherwise stated, significance was set at $p<0.05$.

\section{Results}

Baseline Findings (Before Bariatric Surgery)

The most relevant characteristics of obese and control individuals are displayed in Table 1. Obese participants had, compared to control, decreased spirometric values, higher $\mathrm{FEV}_{1} /$ FVC ratios, and higher serum and exhaled biomarkers but lower adiponectin levels. In obese subjects, comorbidities were common. Eight obese nonsmokers (14\% of total and $24 \%$ of 33 nonsmokers) (median cumulative dose of inhaled mannitol, $176 \mathrm{mg}$ [range, 5-629 mg]) and the same number of obese smokers (8 [14\% of total and $32 \%$ of 25 smokers]) (121 mg [range, 5-475 mg]), respectively, were responsive to mannitol. Responsive obese nonsmokers had, compared to responsive smokers, lower mannitol-induced baseline $\mathrm{FEV}_{1}(2.59 \pm 0.63$ and $3.15 \pm 0.651)$ and final $\mathrm{FEV}_{1}(1.88 \pm$ 0.56 and $2.57 \pm 0.641)$ values, respectively ( $p<0.05$ each), without differences in the rates of AHR (chi-square test) (Table 2). The higher were the exhaled IL-8 levels in obese individuals, the greater were reactivity (RDR) (Rho, 0.35 , $p<0.01$ ) and $\%$ fall $\mathrm{FEV}_{1}$ (Rho, $0.32, p<0.05$ ) to mannitol. AHR was not documented in any of the eight control subjects. There were no differences between obese nonsmokers and smokers who completed the study.

The logistic regression model fitted to data for all obese individuals did not yield interaction (all $p$ values $>0.4$ ), without significant associations with anthropometric co-variables
Table 1 Demographic, clinical, and functional characteristics and serum and exhaled biomarkers in control and obese participants before and after bariatric surgery

Unless otherwise stated, data are expressed as mean $\pm \mathrm{SD}, n(\%)$ or median (interquartile range)

$B M I$ body mass index, OSA obstructive sleep apnea, $M S$ metabolic syndrome, pred predicted, $B D$ bronchodilator, $F V C$ forced vital capacity, $F E V_{l}$ forced expiratory volume in the first second, FRC functional residual capacity, $E R V$ expiratory reserve volume, $s T N F-R 1$ soluble tumor necrosis factor-receptor $1, I L$ interleukin

${ }^{*} p<0.001$ for comparisons between control and obese subjects

?before surgery; ${ }^{* *} p<0.05$ for comparisons between control

?and obese subjects before surgery

\begin{tabular}{|c|c|c|c|c|}
\hline & \multirow[t]{2}{*}{ Control subjects $(n, 20)$} & \multicolumn{2}{|c|}{ Obese subjects $(n, 58)$} & \multirow[t]{2}{*}{$p$ value } \\
\hline & & Before & After & \\
\hline Age, years & $46 \pm 7$ & $46 \pm 12$ & $47 \pm 12$ & - \\
\hline Female, $n(\%)$ & $16(80)$ & $44(76)$ & - & - \\
\hline BMI, $\mathrm{kg} / \mathrm{m}^{2}$ & $22 \pm 3 *$ & $45 \pm 5 *$ & $30 \pm 4$ & $<0.001$ \\
\hline Waist circumference, $\mathrm{cm}$ & $81 \pm 11 *$ & $130 \pm 11 *$ & $97 \pm 11$ & $<0.001$ \\
\hline OSA, $n(\%)$ & - & $41(71)$ & $12(21)$ & $<0.001$ \\
\hline MS, $n(\%)$ & 0 & $44(76)$ & $10(17)$ & $<0.001$ \\
\hline FVC, $\%$ pred & $105 \pm 12 *$ & $90 \pm 13 *$ & $101 \pm 13$ & $<0.001$ \\
\hline $\mathrm{FEV}_{1}, \%$ pred & $103 \pm 13^{* *}$ & $94 \pm 15^{* *}$ & $102 \pm 13$ & $<0.001$ \\
\hline $\mathrm{FEV}_{1} / \mathrm{FVC}, \%$ & $78 \pm 5^{*}$ & $83 \pm 5 *$ & $80 \pm 5$ & $<0.001$ \\
\hline $\mathrm{BD}$ response, $\%$ & $2 \pm 3$ & $5 \pm 6$ & $2 \pm 4$ & $<0.01$ \\
\hline FRC, $\%$ pred & ND & $73 \pm 14$ & $112 \pm 22$ & $<0.001$ \\
\hline ERV, \% pred & ND & $39 \pm 25$ & $113 \pm 32$ & $<0.001$ \\
\hline C-reactive protein, mg/l & $0.4(0.1-0.5)^{*}$ & $6.4(3.7-11.4)^{*}$ & $0.4(0.2-0.8)$ & $<0.001$ \\
\hline Leptin, ng/ml & $13.6(5.6-19.7)^{*}$ & $46.3(38.4-96.9)^{*}$ & $13.6(6.9-22.1)$ & $<0.001$ \\
\hline Adiponectin, $\mu \mathrm{g} / \mathrm{ml}$ & $22.1(18.1-25.9)^{*}$ & $13.4(8.7-18.8)^{*}$ & $19.0(12.0-25.2)$ & $<0.001$ \\
\hline sTNF-R1, ng/ml & $0.4(0.2-0.5)^{*}$ & $1.4(1.0-1.9)^{*}$ & $0.9(0.3-1.3)$ & $<0.001$ \\
\hline IL-8, pg/ml & $2.0(2.0-3.3)$ & $9.4(2.0-31.7)$ & $3.4(1.9-14.2)$ & 0.16 \\
\hline Exhaled IL-8, pg/ml & $0.9(0.4-4.0)^{* *}$ & $4.4(1.5-6.6)^{* *}$ & $4.0(1.1-7.1)$ & 0.62 \\
\hline
\end{tabular}


Table 2 Differences in outcomes between obese subjects with and without airway hyperresponsiveness (AHR) to mannitol before and after bariatric surgery

\begin{tabular}{|c|c|c|c|c|c|c|}
\hline & \multicolumn{2}{|l|}{ Before } & \multirow[t]{2}{*}{$p$ value $^{\mathrm{a}}$} & \multicolumn{2}{|l|}{ After } & \multirow[t]{2}{*}{$p$ value $^{\mathrm{b}}$} \\
\hline & With AHR & Without AHR & & With AHR & Without AHR & \\
\hline Patients, $n$ & 16 & 42 & - & 4 & 54 & - \\
\hline Age, years & $49 \pm 7$ & $46 \pm 12$ & 0.29 & $60 \pm 4$ & $46 \pm 10$ & 0.009 \\
\hline BMI, $\mathrm{kg} / \mathrm{m}^{2}$ & $46 \pm 5$ & $44 \pm 5$ & 0.79 & $27 \pm 3$ & $30 \pm 4$ & 0.11 \\
\hline Waist, cm & $134 \pm 9$ & $128 \pm 11$ & 0.052 & $97 \pm 11$ & $97 \pm 11$ & 0.95 \\
\hline Baseline $\mathrm{FEV}_{1}, \%$ pred & $89 \pm 15^{*}$ & $96 \pm 15$ & 0.15 & $117 \pm 23 * *$ & $99 \pm 13$ & 0.014 \\
\hline Final $\mathrm{FEV}_{1}, \%$ pred & $70 \pm 18^{*}$ & $91 \pm 18$ & $<0.001$ & $97 \pm 17 * *$ & $94 \pm 14$ & 0.79 \\
\hline$\%$ Fall FEV 1 & $21 \pm 9$ & $6 \pm 4$ & $<0.001$ & $16 \pm 1$ & $3 \pm 6$ & $<0.001$ \\
\hline $\mathrm{GM} \mathrm{PD}_{15}, \mathrm{mg}$ & $83(24-145)$ & $>635$ & NA & $224(156-322)$ & $>635$ & NA \\
\hline $\mathrm{RDR}, \% / \mathrm{mg}$ & $0.11(0.05-1.88)$ & $0.007(0.003-0.129)$ & $<0.001$ & $0.05(0.05-0.17)$ & $0.008(0.003-0.011)$ & $<0.001$ \\
\hline Exhaled IL-8, pg/ml & $6.23(2.94-12.66)$ & $3.97(1.26-5.70)$ & 0.99 & $7.10(2.58-11.46)$ & $3.90(1.07-6.51)$ & 0.44 \\
\hline
\end{tabular}

Unless otherwise stated, data are expressed as mean $\pm \mathrm{SD}$ or median (interquartile range); baseline $\mathrm{FEV}_{1}, \mathrm{FEV}_{1}$ values measured after inhaling the 0 -mg of mannitol capsule; final $\mathrm{FEV}_{1}, \mathrm{FEV}_{1}$ values measured at the end of mannitol test; $\%$ fall $\mathrm{FEV}_{1}$, mannitol-induced percentage decrease of $\mathrm{FEV}$ from baseline to final values; $\mathrm{GM}$ (geometric mean) of $\mathrm{PD}_{15}$, provocation dose of mannitol to cause a $15 \%$ fall $\mathrm{FEV}_{1} ; \mathrm{RDR}$, response-dose ratio $\left(\%\right.$ fall $\mathrm{FEV}{ }_{1}$ divided by cumulative dose given of mannitol). For other abbreviations, see Table 1

${ }^{*} p<0.001$ for comparisons between baseline and final $\mathrm{FEV}_{1}$ values before surgery; ${ }^{*} p<0.05$ for comparisons between baseline and final $\mathrm{FEV}_{1}$ values after surgery

${ }^{\mathrm{a}} p$ value for comparisons between obese subjects with and without AHR before surgery

${ }^{\mathrm{b}} p$ value for comparisons between obese subjects with and without AHR after surgery

or cigarette smoking. The levels of exhaled IL-8 had a marginal association with AHR (odds ratio [OR], 1.10; $95 \%$ confidence interval $[\mathrm{CI}], 0.97-1.22 ; p=0.06)$ only.

\section{Findings after Bariatric Surgery}

Sleeve gastrectomy (SG) was performed in 32 and Roux-en-Y gastric bypass (RYGBP) in 26 subjects, without differences in the allocation to the two procedures. The three subjects with $<50 \%$ excess weight loss were nonsmokers, two treated with $\mathrm{SG}$, and one with RYGB. There was an excess weight loss of $76 \pm 17 \%$, and BS was successful (i.e., $>50 \%$ excess weight loss) in 55 participants (95\%). In obese individuals, clinical and spirometric findings and all but serum and exhaled IL-8 biomarkers significantly improved or normalized (Table 1). Two subjects ceased cigarette consumption only.

One year after BS, AHR was abolished in 15 out of the 16 responsive individuals. In addition, three preoperative nonresponsive subjects became now responsive to mannitol. A univariate analysis (McNemar's test) showed a significant reduction in AHR in obese individuals after BS $(p<0.001)$.

The results from the models fitted to all pre- and postoperative data failed to detect any significant interaction (all $p$ values $>0.2$ ). The GEE analysis [31] showed that the period of evaluation before and after BS was the key independent factor associated with AHR improvement (OR, 4.7; $95 \%$ CI $1.4-15.4 ; p<0.01)$. Cigarette smoking was not related to AHR. Likewise, postoperative changes in exhaled IL-8 and in AHR were not significant (OR, 1.09; $95 \%$ CI, 1.0-1.2; $p=0.07$ ).

\section{Discussion}

This is the first prospective study that comprehensively assessed AHR to mannitol in morbidly obese, non-asthmatic adults, including smokers and nonsmokers. Two were the novel findings in our study. First, preoperative AHR to mannitol was abolished after BS in all but one individual and emerged in three new obese individuals. Second, the degrees of airway sensitivity $\left(\mathrm{PD}_{15}\right)$ and reactivity $(\mathrm{RDR})$ to mannitol were considerably altered in obese participants akin to patients with currently active asthma [26, 32]. The prevalence of AHR in obese nonsmokers was high when compared to what has been previously observed in young normal weighted adults [33]. Similarly, our results extend and complement previous studies using direct stimuli in normal weighted individuals $[34,35]$, obese adults with and without asthma [5], and overweight Chinese families of asthmatic adults [36].

The agents that act indirectly to induce AHR are considered more specific for identifying the presence of airway inflammation and more consistent with the diagnosis of asthma than direct stimuli [26]. Numerous hypotheses have been suggested to explain the relationships between obesity, asthma, and AHR, from dietary lifestyle through mechanisms of 
immunoregulation and inflammation [1, 37, 38]. Responsive obese nonsmokers had a higher likelihood of bronchial asthma and might therefore identify patients at high risk of asthma early before the expression of symptoms [10]. Notwithstanding, the mechanisms need to be probed further. From a clinical viewpoint, the significance of a positive response to mannitol is highly suggestive of active bronchial inflammation.

Before surgery, except for serum IL- 8 values, obese individuals had higher biomarkers and lower adiponectin levels than control individuals, indicating more active systemic inflammation [39]. Serum adiponectin levels are reduced in obese subjects, and this is thought to induce systemic complications and augment airway inflammation [7, 19].

There were no differences in the rates of preoperative AHR between obese nonsmokers and smokers, and cigarette smoking was not related to AHR. Some smokers without clinical asthma can also have significant airway sensitivity to mannitol that is lost when smoking is stopped after a few weeks [40]. While the same mechanism of airway inflammation in obese nonsmokers could be invoked in smokers, it is known that smoking also recruits airway macrophages and neutrophils.

Effective weight loss promoted by BS was successfully achieved after surgery in the obese population and was associated to a practical abolition of AHR alone with lung function improvement, including that of most biomarkers and considerable reduction in comorbidities. Moreover, although the current study design did not preclude the role played by other factors that could influence postoperative evaluations potentially linked to changes in AHR, multivariate analysis suggested that BS was a key determinant of the significant AHR improvement observed in the obese cohort. The development of postoperative AHR in three new obese individuals (one nonsmoker and two smokers) and its persistence in one smoker suggest that aging and residual systemic inflammation may have also played an influential role.

The major strength of our study lies in its comprehensive design to assess clinical, functional, and biomarker outcomes in a cohort of asymptomatic, non-asthmatic, morbidly obese individuals with and without smoking habits, before and after surgery. There were, however, two shortcomings. First, the number of obese participants was not high due to the stringent design, and second, the indirect assessment of AHR in the current study was not compared with that made by direct stimuli because it was considered too aggressive in the setting of our study.

In conclusion, baseline preoperative AHR to mannitol in this cohort of asymptomatic morbidly obese candidates to BS is substantially high and associated to systemic inflammation; obese smokers had a similar rate of AHR but not associated to systemic inflammation. Our study provides new insights into the pathophysiology of AHR in obese individuals.

Acknowledgments The authors want to express their gratitude to the patients for their unselfish collaboration, to Lluis Jover, $\mathrm{PhD}$ (Unitat de Bioestadística, Departament de Salut Pública, Universitat de Barcelona) for his expert statistical advice, to John D Brannan, PhD (Sydney, Australia) for his insightful comments, and to Maribel Diaz, PhD (Hospital Clínic), Marc Pino, RT (Hospital Clínic) and Patricia Molina, RT (Hospital Clínic) and Mónica Vilà-Ubach, RT (Hospital del Mar) for the quality of the measurements of systemic and exhaled biomarkers. Likewise, the skillful technical contribution of Conchi Gistau, RN and Conchita Ruiz, RN is graciously acknowledged.

Contributors RRR, JV, and EA contributed to the study design. EA, ER, JV, EB, YT, FB, and RRR contributed to data acquisition. EA, RRR, $\mathrm{JV}$, and EB contributed to analysis and interpretation of data. EA, RRR, $\mathrm{JV}$, and EB contributed to critical revision and drafted the manuscript. All authors approved the final version of the manuscript.

A Conflict of Interest Ebymar Arismendi, Eva Rivas, Josep Vidal, Esther Barreiro, Yolanda Torralba, and Felip Burgos have any conflict of interest with the content of the article. Dr Roberto Rodríguez-Roisin has received a grant-in-aid by Almirall.

A Statement of Informed Consent Informed consent was obtained from all individual participants included in the study.

A Statement of Human and Animal Rights "All procedures performed in studies involving human participants were in accordance with the ethical standards of the institutional and/or national research committee and with the 1964 Helsinki declaration and its later amendments or comparable ethical standards."

Support This study is supported by the Fondo de Investigación Sanitaria (FIS) PI080311, CIBER Enfermedades Respiratorias, the Generalitat de Catalunya (2009SGR00911). and a grant-in-aid by Almirall.

Open Access This article is distributed under the terms of the Creative Commons Attribution License which permits any use, distribution, and reproduction in any medium, provided the original author(s) and the source are credited.

\section{References}

1. Sutherland TJ, Cowan JO, Young S, et al. The association between obesity and asthma: interactions between systemic and airway inflammation. Am J Respir Crit Care Med. 2008;178(5):469-75.

2. Bel EH. Obesity and asthma control. Am J Respir Crit Care Med. 2013;187(7):667-8.

3. Chinn S, Jarvis D, Burney P. Relation of bronchial responsiveness to body mass index in the ECRHS. Eur Community Respir Health Surv Thorax. 2002;57(12):1028-33.

4. Beuther DA, Sutherland ER. Overweight, obesity, and incident asthma: a meta-analysis of prospective epidemiologic studies. Am J Respir Crit Care Med. 2007;175(7):661-6.

5. Shore SA. Obesity, airway hyperresponsiveness, and inflammation. J Appl Physiol. 2010;108(3):735-43. 
6. Sideleva O, Suratt BT, Black KE, et al. Obesity and asthma: an inflammatory disease of adipose tissue not the airway. Am J Respir Crit Care Med. 2012;186(7):598-605.

7. Van Gaal LF, Mertens IL, De Block CE. Mechanisms linking obesity with cardiovascular disease. Nature. 2006;444(7121):875-80.

8. Lumeng CN, Deyoung SM, Bodzin JL, et al. Increased inflammatory properties of adipose tissue macrophages recruited during dietinduced obesity. Diabetes. 2007;56(1):16-23.

9. Brannan JD, Gulliksson M, Anderson SD, et al. Inhibition of mast cell PGD2 release protects against mannitol-induced airway narrowing. Eur Respir J. 2006;27(5):944-50.

10. Anderson SD. Indirect challenge tests: airway hyperresponsiveness in asthma: its measurement and clinical significance. Chest. 2010;138(2 Suppl):25S-30.

11. Buchwald H, Estok R, Fahrbach K, et al. Weight and type 2 diabetes after bariatric surgery: systematic review and meta-analysis. Am J Med. 2009;122(3):248-56.

12. Morinigo R, Casamitjana R, Delgado S, et al. Insulin resistance, inflammation, and the metabolic syndrome following Roux-en-Y gastric bypass surgery in severely obese subjects. Diabetes Care. 2007;30(7):1906-8.

13. Salome CM, Munoz PA, Berend N, et al. Effect of obesity on breathlessness and airway responsiveness to methacholine in non-asthmatic subjects. Int J Obes (Lond). 2008;32(3):502-9.

14. Aaron SD, Fergusson D, Dent R, et al. Effect of weight reduction on respiratory function and airway reactivity in obese women. Chest. 2004;125(6):2046-52.

15. Dixon AE, Pratley RE, Forgione PM, et al. Effects of obesity and bariatric surgery on airway hyperresponsiveness, asthma control, and inflammation. J Allergy Clin Immunol. 2011;128(3):508-15.

16. Boulet LP, Turcotte H, Martin J, et al. Effect of bariatric surgery on airway response and lung function in obese subjects with asthma. Respir Med. 2012;106(5):651-60.

17. Zerah-Lancner F, Boyer L, Rezaiguia-Delclaux S, et al. Airway responsiveness measured by forced oscillation technique in severely obese patients, before and after bariatric surgery. J Asthma. 2011;48(8):818-23.

18. Arismendi E, Rivas E, Vidal J, et al. Airway hyperresponsiveness to mannitol in morbid obesity before and after bariatric surgery. Eur Respir J. 2013;42:4707.

19. Arismendi E, Rivas E, Agusti A, et al. The systemic inflammome of severe obesity before and after bariatric surgery. PLoS One. 2014;9(9):e107859.

20. Jimenez A, Casamitjana R, Flores L, et al. Long-term effects of sleeve gastrectomy and Roux-en-Y gastric bypass surgery on type 2 diabetes mellitus in morbidly obese subjects. Ann Surg. 2012;256(6):1023-9.

21. Moy J, Pomp A, Dakin G, et al. Laparoscopic sleeve gastrectomy for morbid obesity. Am J Surg. 2008;196(5):e56-9.

22. Miller MR, Hankinson J, Brusasco V, et al. Standardisation of spirometry. Eur Respir J. 2005;26(2):319-38.

23. Roca J, Burgos F, Sunyer J, et al. References values for forced spirometry. Group of the European Community Respiratory Health Survey. Eur Respir J. 1998;11(6):1354-62.
24. Roca J, Burgos F, Barbera JA, et al. Prediction equations for plethysmographic lung volumes. Respir Med. 1998;92(3):454-60.

25. Wanger J, Clausen JL, Coates A, et al. Standardisation of the measurement of lung volumes. Eur Respir J. 2005;26(3):511-22.

26. Anderson SD, Brannan J, Spring J, et al. A new method for bronchial-provocation testing in asthmatic subjects using a dry powder of mannitol. Am J Respir Crit Care Med. 1997;156(3 Pt 1):758-65.

27. Munoz PA, Gomez FP, Manrique HA, et al. Pulmonary gas exchange response to exercise- and mannitol-induced bronchoconstriction in mild asthma. J Appl Physiol. 2008;105(5):1477-85.

28. Rodriguez-Trigo G, Zock JP, Pozo-Rodriguez F, et al. Health changes in fishermen 2 years after clean-up of the Prestige oil spill. Ann Intern Med. 2010;153(8):489-98.

29. Horvath I, Hunt J, Barnes PJ, et al. Exhaled breath condensate: methodological recommendations and unresolved questions. Eur Respir J. 2005;26(3):523-48.

30. Muir K, Gomeni R. Pharmacokinetics in drug development: clinical study design and analysis. Non-compartmental analysis. Bonate PL, Howard DR ed. 2004. 235-266.

31. Diggle PJ, Liang KY, Zeger SL. Analysis of longitudinal data. Oxford, United Kingdom: 1994

32. Brannan JD, Koskela H, Anderson SD, et al. Responsiveness to mannitol in asthmatic subjects with exercise- and hyperventilationinduced asthma. Am J Respir Crit Care Med. 1998;158(4):1120-6.

33. Sverrild A, Porsbjerg C, Thomsen SF, et al. Diagnostic properties of inhaled mannitol in the diagnosis of asthma: a population study. $\mathrm{J}$ Allergy Clin Immunol. 2009;124(5):928-32.

34. Malo JL, Pineau L, Cartier A, et al. Reference values of the provocative concentrations of methacholine that cause $6 \%$ and $20 \%$ changes in forced expiratory volume in one second in a normal population. Am Rev Respir Dis. 1983;128(1):8-11.

35. Roth BJ, Hammers LM, Dillard TA. Methacholine challenge testing in Reserve Officer Training Corps cadets. Chest. 2001;119(3):701-7.

36. Celedon JC, Palmer LJ, Litonjua AA, et al. Body mass index and asthma in adults in families of subjects with asthma in Anqing, China. Am J Respir Crit Care Med. 2001;164(10 Pt 1):1835-40.

37. Bullimore SR, Siddiqui S, Donovan GM, et al. Could an increase in airway smooth muscle shortening velocity cause airway hyperresponsiveness? Am J Physiol Lung Cell Mol Physiol. 2011;300(1):L121-31.

38. Sutherland TJ, Cowan JO, Taylor DR. Dynamic hyperinflation with bronchoconstriction: differences between obese and nonobese women with asthma. Am J Respir Crit Care Med. 2008;177(9):970-5.

39. Kumada M, Kihara S, Ouchi N, et al. Adiponectin specifically increased tissue inhibitor of metalloproteinase-1 through interleukin-10 expression in human macrophages. Circulation. 2004;109(17):20469.

40. Stolz D, Anderson SD, Gysin C, et al. Airway reactivity to inhaled mannitol in cigarette smokers: a longitudinal study. Respir Med. 2007;101(7):1470-6. 